\title{
Novi sintaksoni i nomenklaturne preinake u Hrvatskoj (2019.-2020.)
}

\author{
NENAD JASPRICA ${ }^{1 *}$, ŽELJKO ŠKVORC ${ }^{2}$ \\ ${ }^{1}$ Sveučilište u Dubrovniku, Institut za more i priobalje, Kneza Damjana Jude 12, pp. 83, HR-20000 \\ Dubrovnik, Hrvatska
}

${ }^{2}$ Sveučilište u Zagrebu, Šumarski fakultet, Svetošimunska 25, HR-10000 Zagreb, Hrvatska

*Autor za dopisivanje / corresponding author: nenad.jasprica@unidu.hr

Tip članka / article type: kratko stručno priopćenje / short professional communication Povijest članka / article history: primljeno / received: 06.01.2021., prihvaćeno / accepted: 16.01.2021. URL: https://doi.org/10.46232/glashbod.8.2.7

Jasprica, N., Škvorc, Ž. (2020): Novi sintaksoni i nomenklaturne preinake u Hrvatskoj (2019.-2020.). Glas. Hrvat. bot. druš. 8(2): 124-132.

\section{Sažetak}

Na temelju literaturnih podata objavljenih u 2019. i 2020., vegetacija Hrvatske dopunjena je novopisanim i nomenklaturno preinačenim sintaksonima. Novi sintaksoni i preinake utvrđene su unutar razreda Carpino-Fagetea sylvaticae, Nardetea strictae, Molinio-Arrhenatheretea, Erico-Pinetea, Pinetea halepensis, Crithmo-Staticetea, Juncetea maritimi, Asplenietea trichomanis, Cymbalario-Parietarietea diffusae, Phragmito-Magnocaricetea i Dittrichietea viscosae. Za vegetacju Hrvatske opisani su za znanost dva nova razreda (Pinetea halepensisi Dittrichietea viscosae), dva reda (Junipero communis-Pinetalia nigrae i Dittrichietalia viscosae), četiri sveze (Salvio nemorosae-Arrhenatherion, Junipero nanae-Pinion dalmaticae, Capparo orientalis-Aurinion leucadeae i Dittrichion viscosae), 17 asocijacija i 14 subasocijacija. Dodatno, u radu su ukratko prikazane preinake unutar razreda Phragmito-Magnocaricetea.

Ključne riječi: fitocenologija, Hrvatska, klasifikacija, sintaksoni, vegetacija

Jasprica, N., Škvorc, ̌̌. (2020): New syntaxa and nomenclature changes in Croatia (2019-2020). Glas. Hrvat. bot. druš. 8(2): 124-132.

\section{Abstract}

Based on the literature data reviewed in 2019 and 2020, the vegetation of Croatia has been supplemented with newly described and validated syntaxonomic units. New syntaxa and changes were identified within the classes Carpino-Fagetea sylvaticae, Nardetea strictae, Molinio-Arrhenatheretea, Erico-Pinetea, Pinetea halepensis, Crithmo-Staticetea, Juncetea maritimi, Asplenietea trichomanis, Cymbalario-Parietarietea diffusae, Phragmito-Magnocaricetea and Dittrichietea viscosae. For the vegetation in Croatia, two classes (Pinetea halepensis and Dittrichietea viscosae), two orders ((Junipero communis-Pinetalia nigrae and Dittrichietalia viscosae), four alliances (Salvio nemorosae-Arrhenatherion, Junipero nanae-Pinion dalmaticae, Capparo orientalis-Aurinion leucadeae and Dittrichion viscosae), 17 associations and 14 subassociations have been newly described. Additionally, the paper briefly presents changes within the Phragmito-Magnocaricetea class.

Keywords: Croatia, nomenclature, phytosociology, syntaxa, vegetation 
U 2019. i 2020. objavljeni su radovi u kojima je vegetacija Hrvatske dopunjena novopisanim i nomenklaturno preinačenim sintaksonima.

\section{Carpino-Fagetea sylvaticae Jakucs ex Passarge 1968}

U radu Škvorc i sur. (2020a), u okviru razreda mezofilnih i mješovitih listopadnih šuma, opisana je nova asocijacija i dvije subasocijacije bukovih šuma s područja Žumberka i Samoborskog gorja:

Carpino-Fagetea sylvaticae Jakucs ex Passarge 1968 Luzulo-Fagetalia sylvaticae Scamoni et Passarge 1959 Luzulo-Fagion sylvaticae Lohmeyer et Tx. in Tx. 1954

Gentiano asclepiadeae-Fagetum sylvaticaeŠkvorc, Franjić, Krstonošić, et Sever 2020

Fagetalia sylvaticae Pawłowski 1928 Aremonio-Fagion (Horvat 1938) Borhidi in Török et al. 1989

Epimedio-Fagenion Marinček et al. 1993

Hacquetio-Fagetum Ž. Košir ex Borhidi 1963 aceretosum obtusati Škvorc, Franjić, Krstonošić, et Sever 2020

Lamio orvalae-Fagenion Borhidi ex Marinček et al. 1993

Lamio orvalae-Fagetum Horvat 1938

fraxinetosum orni Škvorc, Franjić, Krstonošić, et Sever 2020

\section{Nardetea strictae Rivas Goday et Borja Carbonell in Rivas Goday et Mayor López 1966}

U radu Alegro i sur. (2020), opisana je nova asocijacija za znanost u okviru vegetacije travnjaka tvrdače na sjevernom Velebitu. Autori su asocijaciju pridružili svezi Potentillo montenegrinae-Festucion paniculatae koja prethodno nije bila uvrštena u vegetaciju Hrvatske (usp. Trinajstić 2008, Škvorc i sur. 2017).

Nardetea strictae Rivas Goday et Borja Carbonell in Rivas Goday et Mayor López 1966

Nardetalia strictae Preising 1950
Potentillo montenegrinae-Festucion paniculatae Redžić ex Čarni et Mucina 2015

Festuco bosniacae-Patzkeetum paniculatae Alegro et Šegota 2020

\section{Molinio-Arrhenatheretea Tx. 1937}

U radu Škvorc i sur. (2020b) analizirana je vegetacija travnjaka na dubokim tlima na južnom rubu Panonske nizine (Slovenija, Hrvatska, Srbija). Opisane su nove zajednice, nove kombinacije te izvršena lektotipizacija i neotipizacija sljedećih sintaksona:

Molinio-Arrhenatheretea Tx. 1937

Arrhenatheretalia elatioris Tx. 1931

Salvio nemorosae-Arrhenatherion Škvorc et al. 2020 Filipendulo vulgaris-Arrhenatheretum elatioris Hundt et Hübl ex Ellmauer 1995 trifolietosum montani Škvorc et al. 2020 Botriochloo ischaemi-Arrhenatheretum elatioris Ćuk in Škvorc et al. 2020

Vicio grandiflorae-Arrhenatheretum elatioris Ćuk in Škvorc et al. 2020

Potentillo reptantis-Allietum angulosi Zelnik in Škvorc et al. 2020

Serratulo-Plantaginetum altissimae Ilijanić 1968 [lektotipizirano]

Agrostio-Juncetum conglomerati Šegulja 1977 [lektotipizirano]

Equiseto-Scirpetum sylvatici Šegulja 1977 [lektotipizirano] Ventenato-Trifolietum pallidi Ilijanić 1968 [lektotipizirano]

Succisello inflexae-Deschampsietum cespitosae (Hayek ex Horvatić 1930) Ellmauer in Ellmauer et Mucina 1993

sanguisorbetosum officinalis Zelnik in Škvorc et al. 2020

Bromo racemosi-Cynosuretum cristati Horvatić et Tomažič 1941 [lektotipizirano] danthonietosum Šegulja 1977 [lektotipizirano] Agrostio caninae-Hordeetum secalini Ilijanić in Horvatić, Ilijanić et Marković-Gospodarić 1970 [tipizirano] 

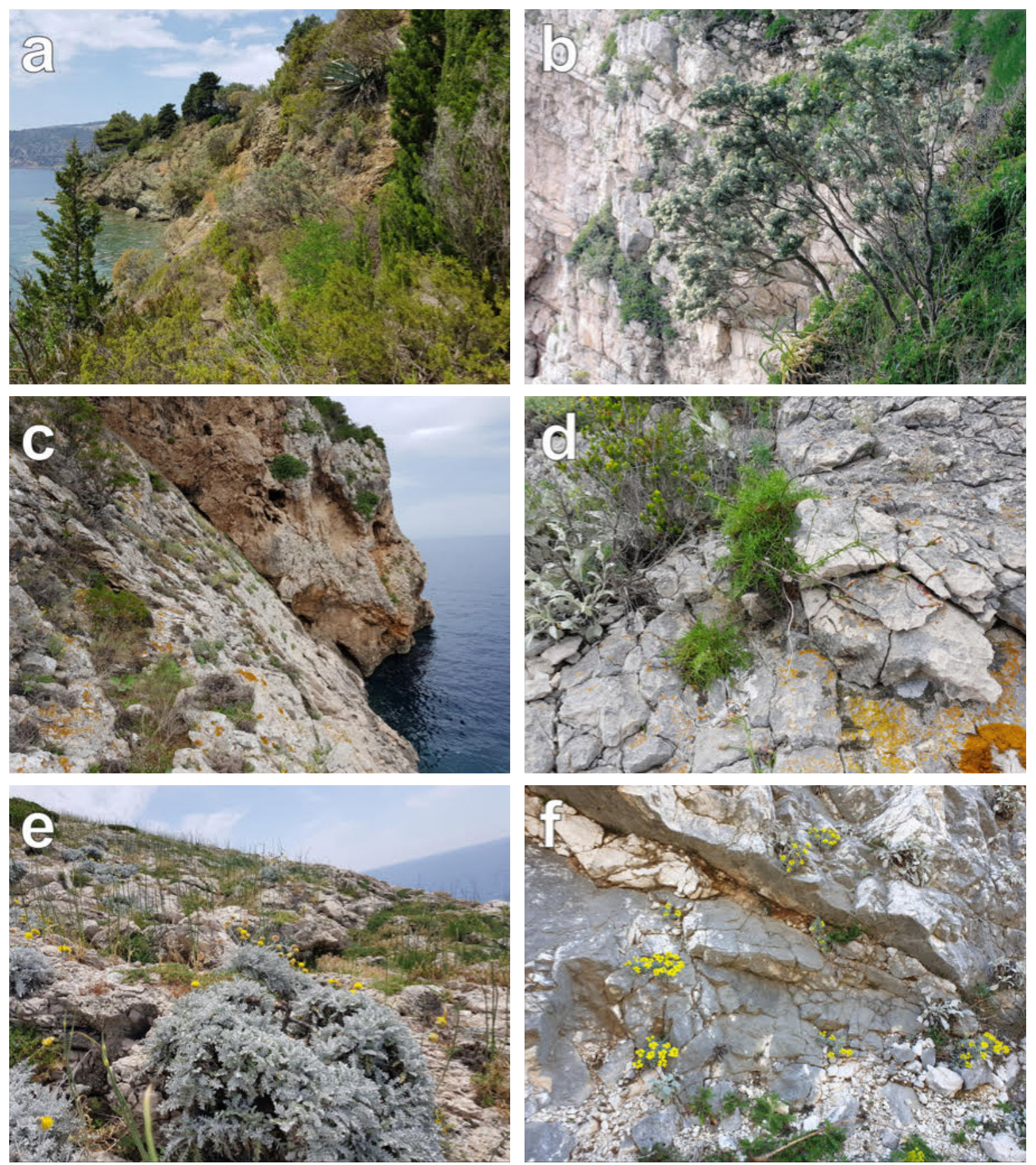

Slika 1. Halotolerantna vegetacija sveze Anthyllidion barbae-jovis i zajednica s Anthyllis barba-jovis u Komiži na otoku Visu (A) i na otoku Koločepu (B), sveza Capparo orientalis-Aurinion leucadeae (C) te detalj asocijacije Pimpinello lithophyllae-Centaureetum issaeae na Visu (D). Vegetacija Centaureetum ragusinae limonietosum cancellati na sv. Andriji, Viški arhipelag (E) i Moltkio petraeae-Inuletum verbascifoliae fibigietosum triquetrae iznad Omiša (F) (foto N. Jasprica). 
Ranunculo repentis-Alopecuretum pratensis (Eggler 1933) Ellmauer1993 deschampsietosum Škvorc et al. 2020 hypochoeridetosum radicatae (Hruška-Dell' Uomo 1976) Škvorc et al. 2020

Ranunculo acris-Alopecuretum pratensis $\mathrm{M}$. Vučković ex Aćić et al. 2013

filipenduletosum vulgaris Škvorc et al. 2020

Trifolio pallidi-Alopecuretum pratensis Cincović 1959

lysimachietosum nummulariae Ćuk in Škvorc et al. 2020

Trifolio-Agrostietum stoloniferae Marković 1978 [lektotipizirano] juncetosum effusi Škvorc et al. 2020

Rumici crispi-Agrostietum stoloniferae Moor 1958 menthetosum (Marković 1978) Škvorc et al. 2020

\section{Erico-Pinetea Horvat 1959}

Biondi i Allegrezza (2020) su opisali novi red i svezu u koju su uvrstili i naše šume dalmatinskog crnog bora s Biokova. Sintaksonomski položaj šume dalmatinskog crnog bora s Biokova je:

\section{Erico-Pinetea Horvat 1959}

Junipero communis-Pinetalia nigrae Biondi et Allegrezza 2020

Junipero nanae-Pinion dalmaticae Biondi et Allegrezza 2020

Junipero sibiricae-Pinetum dalmaticae (Domac 1956) 1965

\section{Pinetea halepensis Bonari et Chytrý 2020}

U radu Bonari i sur. (2020), istraživane su sredozmene termofilne borove šume u kojima dominiraju Pinus brutia, P. halepensis, $P$. pinaster i P. pinea. U Jadranskom bazenu šume alepskog bora pripadaju novoopisanom razredu Pinetea halepensis s redom koji je prethodno bio uključen u vegetaciju primorskih vazdazelenih šuma i makija Quercetea ilicis
Br.-Bl. ex A. Bolós et O. de Bolòs in A.Bolòs y Vayreda 1950 (v. Mucina i sur. 2016, Škvorc i sur. 2017):

Pinetea halepensis Bonari et Chytrý 2020

Pinetalia halepensis Biondi, Blasi, Galdenzi, Pesaresi et Vagge in Biondiet al. 2014

Pistacio lentisci-Pinion halepensis Biondi, Blasi, Galdenzi, Pesaresi et Vagge in Biondi et al. 2014 Pistacio lentisci-Pinetum halepensis De Marco, Veri et Caneva 1984

\section{Crithmo-Staticetea Br.-Bl. in Br.-Bl. et al. 1952}

U radu Terzi i sur. (2020) opisano je nekoliko novih sintaksona na otocima Viškog arhipelaga. U okviru vegetacije na stijenama u zoni prskanja mora unutar sveze Limonion anfracti-cancellati opisano je pet novih asocijacija.

Po prvi put za Hrvatsku i istočnu obalu Jadrana, detaljno je opisana zajednica s Anthyllis barba-jovis, koja je uvrštena, u svezu Anthyllidion barbae-jovis i red Helichrysetalia italici, sukladno Škvorc i sur. (2017). Do sada su zajednice s Anthyllis barba-jovis pripisivane hazmofitskoj vegetaciji stijena reda Centaureo-Campanuletalia (Horvatić 1971, Trinajstić 1995). Redu Helichrysetalia italici pripada halotolerantna vegetacija polugrmova na stijenama između dijela obale koja je pod izravnim utjecajem prskanja mora (halofitske zajednice grebenjača Limonion anfracti-cancellati) i hazmofitske vegetacije stijena (Centaureo-Campanuletalia).

Istovremeno, opisana je nova sveza Capparo orientalis-Aurinion leucadeae, endemična za srednjejadransko otočje, uključujući Palagružu i Tremite (Italija). Sveza uključuje hazmofitske zajednice koje nisu izravno izložene prskanju mora „ali su u svakom slučaju pod takvim utjecajem tijekom jakih oluja na Jadranu“ (Lovrić i Rac 1991, Bertović i Lovrić 1992). Unutar te sveze opisane su četiri nove asocijacije, od toga su tri nove za Hrvatsku.

Sintaksonomski položaj zajednica je kako slijedi: 
Crithmo-Staticetea Br.-Bl. in Br.-Bl. et al. 1952

Crithmo-Staticetalia Molinier 1934

Limonion anfracti-cancellati (Horvatić 1934)

Mucina in Mucina et al. 2106

Crithmo maritimi-Limonietum busiani Terzi, Bogdanović, D’Amico et Jasprica 2020 [Biševo]

Crithmo maritimi-Limonietum brusnicensae

Terzi, Bogdanović, D’Amico et Jasprica 2020

[Brusnik]

Crithmo maritimi-Limonietum issaeae Terzi, Bogdanović, D’Amico et Jasprica 2020 [Vis]

Crithmo maritimi-Limonietum pomoense

Trinajstić corr. Terzi, Bogdanović, D’Amico et Jasprica 2020 [Jabuka]

Plantagino holostei-Limonietum subanfracti Terzi, Bogdanović, D’Amico et Jasprica 2020 [Vis]

Helichrysetalia italici Biondi et Géhu in Géhuet Biondi 1994

Anthyllidion barbae-jovis S. Brullo et DeMarco 1989

Anthyllis barba-jovis community [Komiža, Vis]

Capparo orientalis-Aurinion leucadeae Lovrić ex Terzi, Bogdanović, D’Amico et Jasprica 2020

Centaureo jabukensis-Aurinietum leucadeae

Terzi, Bogdanović, D’Amico et Jasprica 2020 [Jabuka]

Limonio vestiti-Puccinellietum teyberi Terzi, Bogdanović, D’Amico et Jasprica 2020 [Kamik]

Pimpinello lithophyllae-Centaureetum issaeae Terzi, Bogdanović, D’Amico et Jasprica 2020 [Vis]

Centaureo diomedeae-Aurinietum leucadeae Terzi, Bogdanović, D’Amico et Jasprica 2020 [Otočje Tremiti, Italija]

\section{Juncetea maritimi Br.-Bl. in Br.-Bl. et al. 1952}

U okviru vegetacije primorskih sitina i halonitrofilnih travnjaka, Dítě i sur. (2019) opisali su novu asocijaciju za znanost Limonio narbonensis-Caricetum divisae u ornitološkom rezervatu Kolansko blato na Pagu. Sintaksonomski položaj je:

Juncetea maritimi Br.-Bl. in Br.-Bl. et al. 1952 Juncetalia maritimi Br.-Bl. ex Horvatić 1934 Juncion maritimi Br.-Bl. ex Horvatić 1934

Limonio narbonensis-Caricetum divisae Z. Dítě, Šuvada, Eliáš, Píš et D. Dítě 2019

\section{Asplenietea trichomanis (Br.-Bl. in Meier et Br.-Bl. 1934) Oberd. 1977}

U radu Terzi i Jasprica (2020) revidirana je hazmofitska vegetacija sveze Centaureo cuspidatae-Portenschlagiellion ramosissimae Trinajstić ex Terzi et Di Pietro 2016 (obuhvaća tertitorije Hrvatske, Bosne i Hercegovine i Crne Gore).

U okviru asocijacije Centaureetum ragusinae Horvat ex Terzi, Jasprica et Caković 2017, opisana je nova subasocijacija C.r. limonietosum cancellati Terzi et Jasprica 2020.

U okviru asocijacije Moltkio petraeae-Inuletum verbascifoliae opisane su tri nove subasocijacije uz dvije promijenjene statusa:

Moltkio petraeae-Inuletum verbascifoliae Wendelberger in Lovrić et Rac 1987 [=Fibigio triquetrae-Cerinthetum tristis Lovrić in Lovrić et Rac 1987, Inulo verbascifoliae-Centaureetum cuspidatae Trinajstić ex Terzi et Di Pietro 2016, Portenschlagiello ramosissimae-Cam panuletum portenschlagianae Trinajstić ex Jasprica et Terzi 2017]:

typicum Terzi et Jasprica 2020

centaureetosum cuspidatae (Terzi et Di Pietro 2016)

Terzi et Jasprica 2020 [=Inulo verbascifoliae-Centaureetum cuspidatae Trinajstić ex Terzi et Di Pietro 2016]

fibigietosum triquetrae (Jasprica et Terzi 2017) Terzi et Jasprica 2020 [=Portenschlagiello ramosissimae-Campanuletum portenschlagianae Trinajstić ex Jasprica et Terzi 2017]

\section{Cymbalario-Parietarietea diffusae Oberd. 1969}

U radu Jasprica i sur. (2020), opisane su tri nove asocijacije za znanost unutar hazmofitske vegetacije stijena, od kojih su dvije nove za Hrvatsku. Uz to, po prvi put za istočnu obalu Jadrana (sv. Andrija, Viški arhipelag) opisana je zajednica Artemisia arborescens-Coronilla valentina koja je priključena svezi Artemisio arborescentis-Capparidion spinosae. Sintaksonomski položaj novoopisanih sintaksona je sljedeća (*AL - Albanija, HR - Hrvatska, HU - Mađarska, MNE - Crna Gora, NMK - Sjeverna Makedonija, SI - Slovenija): 
Cymbalario-Parietarietea diffusae Oberd. 1969

Tortulo-Cymbalarietalia Segal 1969

Cymbalario-Asplenion Segal 1969

Asplenio rutae-murario-Campanuletum rotundifoliae Jasprica, Škvorc et Purger 2020 [HU, SI]* zajednica Asplenium trichomanes-Arabis hirsuta [HR]

Galio valantiae-Parietarion judaicae Rivas-Mart. ex de Bolòs 1967

Sedo dasyphilli-Campanuletum austroadriaticae Jasprica, Škvorc et Kovačić 2020 [HR, MNE, AL] Soncho tenerrimi-Parietarietum judaiace Jasprica, Škvorc, Pandža et Milović 2020 [HR, MNE, NMK]

zajednica Antirrhinum majus-Capparis orientalis [HR, MNE]

Artemisio arborescentis-Capparidion spinosae Biondi, Blasi et Galdenzi in Biondi et al. 2014 zajednica Artemisia arborescens-Coronilla valentina [Viški arhipelag, HR]

\section{Phragmito-Magnocaricetea Klika in Klika et Novák 1941}

U radu Landucci i sur. (2020), objavljena je klasifikacija močvarne vegetacije (tršćaci, rogozici i šašici) u Europi. Predložena je izmjena sintaksonomske klasifikacije objavljene u "EuroVegChecklist" (Mucina i sur. 2016, Škvorc i sur. 2017). Većina novih naziva asocijacija odnosi se na asocijacije koje su ranije opisane u literaturi, ali ne i u skladu s „International Code of Phytosociological Nomenclature" (Weber i sur. 2000).

Sveza Phalaridion arundinaceae Kopecký 1961 isključena je iz reda Nasturtio-Glycerietalia Pignatti 1953 jer joj nedostaju dijagnostičke vrste. Predložena je samo jedna asocijacija s Phalaris arundinacea $\mathrm{L}$. (Phalaridetum arundinaceae Libbert 1931), a uvrštena je u svezu Phragmition communis Koch 1926 (Phragmitetalia Koch 1926) zbog relativno visoke učestalosti vrsta iz te sveze. Zaključeno je kako se ranije dvije opisane asocijacije (i) Phalaridetum arundinaceae Libbert 1931 (Magnocaricion gracilis
Géhu 1961) te (ii) Rorippo-Phalaridetum arundinaceae Kopecký 1961 (Phalaridion arundinaceae Kopecký 1961) floristički i ekološki ne razlikuju.

Opisana je nova sveza Bolboschoeno maritimi-Schoenoplection tabernaemontani Landucci et al. 2020 (nomenklaturni tip Schoenoplectetum tabernaemontani Soó 1947). Standardizirani su nazivi asocijacija u kojoj dominira Sium latifolium L. kao Glycerio maximae-Sietum latifolii Landucci et al. 2020 te Veronica beccabunga L. kao Glycerio notatae-Veronicetum beccabungae Landucci et al. 2020.

\section{Dittrichietea viscosae Trinajstić, B.Foucault et Jasprica 2019}

U radu Foucault i Jasprica (2019) izvršena je nomenklaturna preinaka razreda Dittrichietea viscosae Trinajstić, B.Foucault et Jasprica 2019. Taj razred je kao "vegetaciju napuštenih poljoprivrednih površina ("nerizi”) Sredozemlja sa skeletnim smeđim, primorskim tlom izgrađenoj pretežito od polugrmovitih elemenata (kamefita)" izvorno opisao Trinajstić (1965) u svojoj doktorskoj disertaciji, ali kasnije nije validno objavljen (Trinajstić 1978, 1992) te je, kao takav, uvršten u djelo Biljne zajednice Republike Hrvatske (Trinajstić 2008) pod nazivom Inuletea viscosae Trinjastić (1965) 1978, s redom Inuletalia viscosae Trinjastić (1965) 1978 i svezom Inulion viscosae Trinjastić (1965) 1978. U svezu su izvorno uključene dvije asocijacije Helichryso-Inuletum viscosae Trinajstić 1965 te Dauco majoris-Foeniculetum vulgaris Trinajstić 2008 (Trinajstić 2008). U “EuroVegChecklist” (Mucina i sur. 2016, Škvorc i sur. 2017), ovaj je tip vegetacije uvršten unutar razreda ruderalne vegetacije visokih zeleni na suhim staništima Artemisietea vulgaris Lohmeyer et al. in Tx. ex von Rochow 1951, odnosno unutar reda i sveze sredozemnih ruderalnih zajednica na zapuštenim površinama Elytrigio repentis-Dittrichietalia viscosae Mucina 2016 i Inulo viscosae-Agropyrion repentis Biondi et Allegrezza 1996. Nakon toga su Di Pietro i sur. (2017) opisali asocijaciju Helichryso italici-Dittrichietum viscosae 

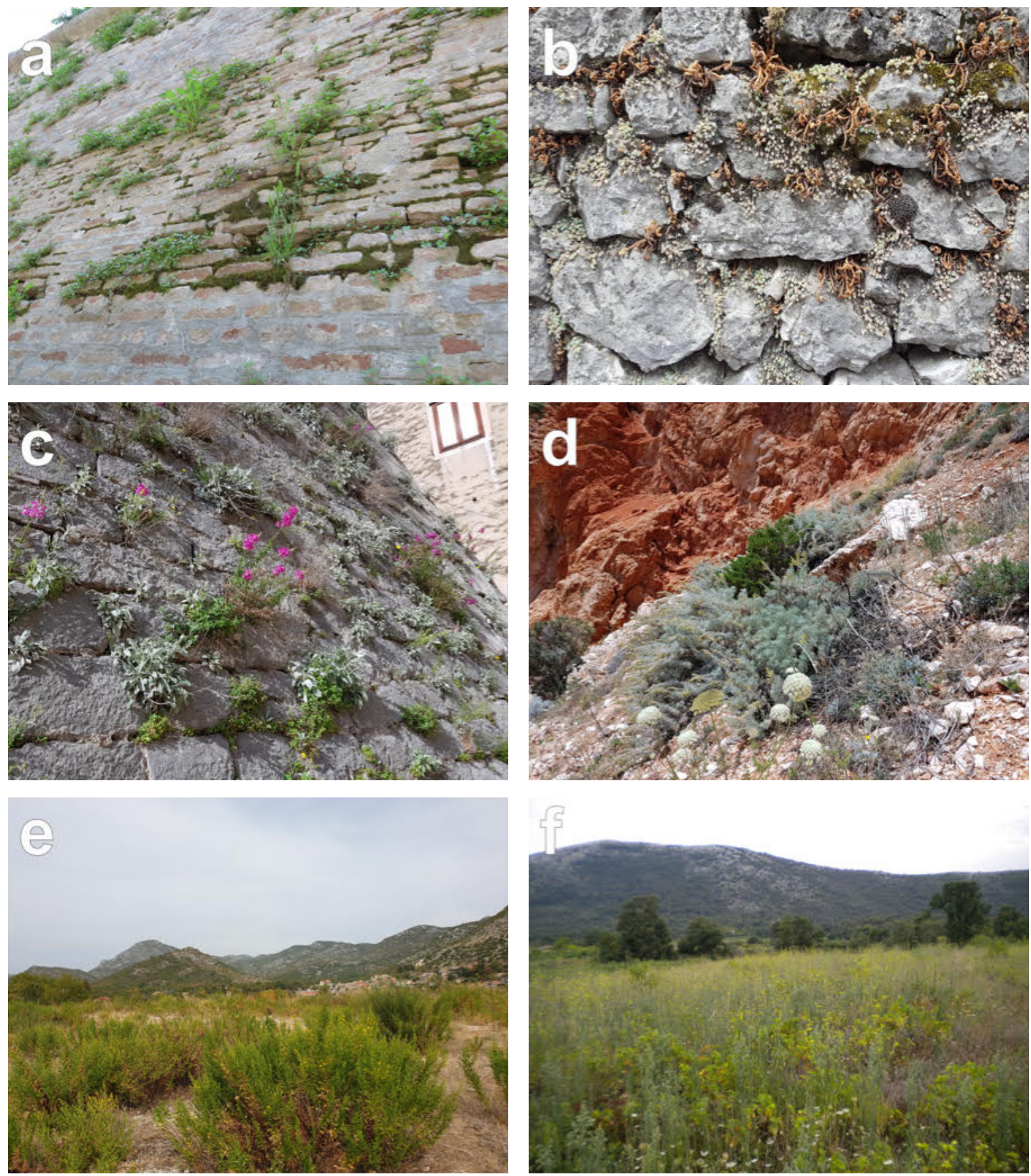

Slika 2. Asocijacija Soncho tenerrimi-Parietarietum judaicae u Zadru (A), detalj asocijacije Sedo dasyphilli-Campanuletum austroadriaticae na tvrđavi Rozafa, Shkodër, Albanija (B), detalj zajednice Antirrhinum majus-Capparis orientalis u Splitu (C), vegetacija sveze Artemisio arborescentis-Capparidion spinosae (zajednica Artemisia arborescens-Coronilla valentina) na sv. Andriji, Viški arhipelag (D), Loto hirsuti-Dittrichietum viscosae u mjestu Gromača u Dubrovačkom primorju (E) i Dauco majori-Foeniculetum vulgaris u Potomju na Pelješcu (F) (foto N. Jasprica). 
Trinajstić ex Di Pietro, Germani et Fortini 2017 i priključili je svezi Linarion purpureae S. Brullo 1984, redu Scrophulario-Helichrysetalia Brullo 1984 i razredu Drypidetea spinosae Quézel 1964.

Sintaksonomski položaj prema navodu u Foucault i Jasprica (2019) je sljedeći:

Dittrichietea viscosae Trinajstić, B.Foucault et Jasprica 2019

Dittrichietalia viscosae Trinajstić, B.Foucault et Jasprica 2019

Dittrichion viscosae Trinajstić, B.Foucault et Jasprica 2019

Loto hirsuti-Dittrichietum viscosae Trinajstić, B.Foucault et Jasprica 2019

\section{Literatura}

Alegro, A., Šegota, V., Vuković, N., Kabaš, E., Lakušić, D. (2020): Patzkea paniculata grasslands in the north-western Dinarides - a crossroad between the Alps and the Balkan mountains. Phytocoenologia 50: 279-295.

Bertović, S., Lovrić, A.Ž. (1992): Übersicht der Vegetation Kroatiens nach neueren Untersuchungen. Tuexenia12: 29-48.

Biondi, E., Allegrezza, M. (2020): Syntaxonomy of Pinus nigra s.l. communities in the Erico-Pinetea class and their distribution in the central Apennines and Balkan province. Plant Biosystems 154: 248-258.

Bonari, G., Fernández-González, F., Çoban, S., Monteiro-Henriques, T., Bergmeier, E., Didukh Ya, P., Xystrakis, F., Angiolini, C., Chytrý, K., Acosta, A.T.R., Agrillo, E., Costa, J.C., Danihelka, J., Hennekens, S.M., Kavgac1, A., Knollová, I., Neto, C.S., Sağlam, C., Škvorc, Ž., Tichý, L., Chytrý M. (2020): Classification of the Mediterranean lowland to submontane pine forest vegetation. Applied Vegetation Science. DOI: 10.1111/avsc.12544

Di Pietro, R., Germani, D., Fortini, P. (2017): A phytosociological investigation on the mixed hemycryptophitic and therophitic grasslands of the Cornicolani mountains (Lazio Region - central Italy). Plant Sociology 54: 107-128.

Foucault, B. (de), Jasprica, N. (2019): Sur une classe de végétation méconnue en France: les Dittrichietea viscosae Trinajstić, B.Foucault et Jasprica classis nova. Botanique 5: 155-170.

Horvatić, S. 1971. Osnovne vegetacijske jedinice primorskog krša i pitanje njihove pojačane zaštite. In: Varićak, T. (ur.) Simpozij o zaštiti prirode u našem kršu, 109-135. Jugoslavenska akademija znanosti i umjetnosti, Zagreb.

Jasprica, N., Škvorc, Ž., Pandža, M., Milović, M., Purger, D., Krstonošić, D., Kovačić, S., Sandev, D., Lasić, A., Caković, D., Anđić, B., Vujačić Stanišić, M. (2020): Phytogeographic and syntaxonomic diversity of wall vegetation (Cymbalario-Parietarietea diffusae) in southeastern Europe. Plant Biosystems, DOI:10.1080/11263504.2020.1762794

Landucci, F., Šumberová, K., Tichý, L., Hennekens, S., Aunina, L., Biță-Nicolae, C., Borsukevych, L., Bobrov, A., Čarni, A., De Bie, E., Golub, V., Hrivnák, R., Iemelianova, S., Jandt, U., Jansen, F., Kącki, Z., Lájer, K., Papastergiadou, E., Šilc, U., Sinkevičienè, Z., Stančić, Z., Stepanovič, J., Teteryuk, B., Tzonev, R., Venanzoni, R., Zelnik, I., Chytrý M. (2020): Classification of the European marsh vegetation (Phragmito-Magnocaricetea) to the association level. Applied Vegetation Science 23: 297-316.

Lovrić, A., Rac, M. (1991): A comparison of the coastal vegetation of Greece, Yugoslavia, Bulgaria and Turkey. Botanika Chronika 100: 315-324.

Mucina, L., Bültmann, H., Dierßen, K., Theurillat, J.-P., DenglerJ., Čarni, A., Šumberová, K., Raus, T., Di Pietro, R., Gavílan García, R., Chytrý, M., Iakushenko, D., Schaminée, J.H.J., Bergmeier, E., Santos Guerra, A., Daniëls, F.J.A., Ermakov, N., Valachovic, M., Pignatti, S., Rodwell, J.S., Pallas, J., Capelo, J., Weber, H. E., Lysenko, T., Solomesh, A., Dimopolous, P., Aguiar, C., Freitag, H., Hennekens, S.M., Tichý, L., 2016: Vegetation of Europe: Hierarchical floristic classification system of plant, lichen, and algal communities. Applied Vegetation Science 19 (Suppl. 1): 3-264.

Škvorc, Ž., Ćuk, M., Zelnik, I., Franjić, J., Igić, R., Ilić, M., Krstonošić, D., Vukov, D., Čarni, A. (2020b): Diversity of wet and mesic grasslands along a climatic 
gradient on the southern margin of the Pannonian Basin. Applied Vegetation Science 23: 676-697.

Škvorc, Ž., Franjić, J., Krstonošić, D., Sever, K. (2020a): Phytosociological analysis of beech forests in the Žumberak and Samobor highlands (Croatia). Hacquetia, DOI: 10.2478/hacq-2020-0013

Terzi, M., Bogdanović, S., D'Amico F.S., Jasprica, N. (2020): Rare plant communities of the Vis Archipelago (Croatia). Botany Letters 16: 241-254. Terzi, M., Jasprica, N. (2020): Syntaxonomic revision of chasmophytic vegetation of the Centaureo cuspidatae-Portenschlagiellion ramosissimae (Southern Adriatic). Plant Biosystems 154: 9-16.

Trinajstić, I. (1965): Vegetacija otoka Krka. Doktorska disertacija. Prirodoslovno-matematički fakultet, Sveučilište u Zagrebu. 702 pp.
Trinajstić, I. (1978): Razred Inuletea viscosae Trinajstić class nov. In: Gaži Baskova, V., Lovašen-Eberhardt, Ž., Šegulja, N., Šugar, I., Trinajstić, I. (ur.), Tumač uz vegetacijsku kartu list Pula 1:100.000, 38-39. Botanički zavod Prirodoslovno-matematičkog fakulteta Sveučilišta u Zagrebu, Zagreb.

Trinajstić, I. (1992): Vegetacija otoka Unija. Otočki ljetopis 8: 81-94. Mljeta. In: Durbešić, P., Benović, A. (ur.), Mljet, Priopćenja sa simpozija "Prirodne značajke i društvena valorizacija otoka Mljeta”. 247-269. Ekološke monografije 6. Hrvatsko ekološko društvo, Zagreb. Trinajstić, I. (2008): Biljne zajednice Republike Hrvatske. Akademija šumarskih znanosti, Zagreb. 